\title{
Improving drug chart documentation in elective surgical patient admissions.
}

\author{
Alice Thompson \\ Croydon University Hospital
}

\begin{abstract}
National Institute for Health and Care Excellence (NICE) guidelines state that all healthcare organisations should put policies in place for medication reconciliation on admission. At Croydon University Hospital a medication history had previously been taken in Foundation Year 1 (FY1) preoperative clinics. However, when these clinics were deemed unnecessary, this opportunity for drug chart documentation was lost, along with an awareness of this responsibility among the FY1s. As a result patients were being admitted to wards without adequate drug chart documentation which resulted in a serious untoward incident occurring. This project aimed to increase awareness among FY1s of this responsibility and as a result increase drug chart documentation in postoperative elective surgical patients.
\end{abstract}

The drug charts of 40 postoperative elective surgical patients admitted to all surgical wards were reviewed over a 2 week period. 12.5\% (4/32) of patients taking regular medication had these correctly prescribed, with 'high risk medication omissions' found in 7.5\% (3/40). Documentation of an allergy status was absent in $17.5 \%(7 / 40)$ of patients, including $5 \%(2 / 40)$ of whom had a known drug allergy.

To create awareness of this responsibility, first, a presentation was given to the FY1s, second, posters to prompt action were placed on the elective surgical wards, and finally, the Director of Medical Education emailed the FY1s reiterating these facts. We then reviewed the drug charts of 45 elective postoperative patients over a 2 week period following these interventions.

The results showed: correct prescription of regular medications improved to $48 \%$ (16/33); 'high risk medication omissions' reduced to $4 \%$ (2/45); documentation of allergy status on the drug charts increased to $87 \%$ (39/45); failure to document a known drug allergy on the drug chart fell to $2 \%(1 / 45)$; and patients with a 'high risk medication omission' or an undocumented known drug allergy decreased from $12.5 \%$ $(5 / 40)$ to $7 \%(3 / 45)$.

This study has highlighted an area in which medication reconciliation and drug chart documentation were inadequate, and posed a risk to patient safety. Interventions designed to educate the FY1s and inform them of their responsibility improved standards in regular medication prescription and allergy documentation. However, leaving the onus with the FY1s was not enough to achieve adequate drug chart documentation. Further project cycles may therefore require the implementation of a step within the patient admission protocol in the preoperative ward that requires the nursing staff to contact the team's doctor when the patient arrives in hospital to ensure satisfactory drug chart documentation. In addition, collaboration with the pharmacists could also allow a 'best possible medication history' to be taken on the day of admission and thus reduce risk to patient safety.

\section{Problem}

A 65-year-old nursing home resident who underwent an elective laparoscopic cholecystectomy was admitted to an elective surgical ward at 20:00 in February 2013. Thromboprophylaxis and analgesia were prescribed on her drug chart; however, intravenous fluids and regular medications, including her antiepilepetic medication, were not prescribed. She was reviewed at 08:00 the next morning on the ward round, when she was difficult to rouse. The differential diagnoses included a post-ictal state and pre-renal acute renal failure, resulting from drug chart omissions.

In previous years at Croydon University Hospital (CUH), Foundation Year 1 doctors (FY1s) have attended preoperative assessment clinics to clerk patients, take a primary medication history, and prescribe regular medications on patients' drug charts. Last year an audit was performed which revealed that few FY1 assessments were ever read again. Ultimately, these clinics were deemed unnecessary and cancelled. As a result, this opportunity for drug chart documentation was lost, along with an awareness of this responsibility among the FY1s. This could lead to a situation where, following their operation, patients might only have had postoperative medications prescribed on the drug chart by the anaesthetists (typically analgesia and anti-emetics). As many FY1s were not aware it was their responsibility to fill out the patients' drug charts on admission to the hospital, patients were being transferred to wards from theatres without adequate drug chart documentation of allergies and regular medication.

\section{Background}

National Institute for Health and Care Excellence (NICE) guidelines state that all healthcare organisations should put policies in place for medicines reconciliation on admission (1). The World Health 
Organization (WHO) also recommend that healthcare organisations must "maintain accurate medication information" (2). Medication reconciliation by pharmacists typically utilises the 'best possible medication history' (BPMH) which includes an initial interview with a patient or their family and verifying this with at least one other reliable source of information. However, ward based patients only have their drug charts reviewed by a pharmacist specifically for BPMH in daytime working hours. Studies have shown that over one third of patients have medications discrepancies on admission to hospital (3).

Elective surgical patients frequently enter hospital via a preoperative ward and are then taken to operating theatre, recovery, and finally admitted to the elective surgical wards, following their operation. Their arrival onto the surgical wards is therefore often outside pharmacist working hours, and so no BPMH would be achieved until the following morning. This gap is normally bridged by a healthcare professional taking a primary medication history; however, as previously mentioned, an awareness of this responsibility had been lost.

This project aimed to increase awareness among FY1s of the responsibility to take a primary medication history, as with any person being admitted to hospital, and to accurately prescribe appropriate medications and document allergy status on their drug chart. As a consequence we hoped to increase drug chart documentation in postoperative elective surgical patients.

\section{Baseline Measurement}

The target population included all elective postoperative patients admitted to all the surgical wards at CUH over a 2 week period in February 2013. A short proforma was used to assess thromboprophylaxis prescription, allergy status documentation, and postoperative analgesia and to determine whether regular medications had been prescribed. Particular focus was placed on antiepileptic medications, insulin, HAART (highly active antiretroviral therapy), and steroids which were put into a 'high risk medication omission' category and assessed with specific targeted questions addressed to the patient.

Data were collected from 40 patients over a 2 week period. Only $12.5 \%(4 / 32)$ of the patients who took regular medication had any of these medications prescribed. $7.5 \%(3 / 40)$ of all 40 patients had 'high-risk medication omissions'. Furthermore, 17.5\% (7/40) had no documentation of allergy status on the drug chart; of this group $28.6 \%(2 / 7)$ had a known allergy $(5 \%$ of the total number of patients). Patients with a high risk omission, including the 'high risk prescription omissions' or an undocumented known drug allergy, totalled $12.5 \%(5 / 40)$.

See supplementary file: ds2263.pptx - "Pre-intervention Results"

\section{Design}

To improve awareness among the FY1s and inform them of their responsibilities, including taking a medicines history on admission, and subsequent drug chart documentation, three interventions were implemented. First, baseline data were presented at the FY1 handover meeting as junior doctors swapped to new rotations. The scenarios where omission of medications had resulted in a serious untoward incident were presented, along with how the loss of FY1 preoperative clinics had also meant the writing of drug charts had been lost. Ultimately, it was explained that the responsibility of postoperative drug chart documentation still lay with the surgical FY1s. This could be achieved by examining the theatre lists from that day and documenting allergies and regular medications on the drug chart on the day of admission. It was accepted that there are situations where completion of this by the day team is difficult and in these situations the task should be handed over, and completed, by the surgical FY1 on call. Posters were also distributed on the elective surgical wards to prompt action by FY1s, and nursing teams on these wards were alerted to these and encouraged to contact the FY1 on call to complete the job if not done by the day team. Finally, the Director of Medical Education emailed the FY1s directly, reiterating these points to demonstrate support from the faculty on this initiative.

\section{Post-Measurement}

One month after these interventions were made, a repeat survey was performed over a 2 week period in May 2013. The drug charts of 45 elective postoperative patients were reviewed. Correct prescription of patients' regular medications improved from 12.5\% (4/32) to $48 \%$ (16/33). 'High risk medication omissions' reduced from $7.5 \%(3 / 40)$ to $4 \%(2 / 45)$. Allergy status documentation on the drug chart improved from $82.5 \%(33 / 40)$ to $87 \%$ (39/45). Failure to document a known drug allergy on the drug chart reduced from $5 \%$ $(2 / 40)$ to $2 \%(1 / 45)$. In total, patients with 'high risk medication omissions' or an undocumented known drug allergy decreased from $12.5 \%(5 / 40)$ to $7 \%(3 / 45)$. Initial results showed an increase in prescription of regular medication, fewer omissions of known drug allergies on the drug chart, and fewer omissions of high risk medications.

See supplementary file: ds2264.pptx - "Post-intervention Results"

\section{Lessons and Limitations}

The greatest limitation to the implementation of this pathway was the need to persuade the FY1s to increase their workload. The basic interventions that were attempted to increase awareness made some improvement to postoperative drug chart documentation, although leaving the onus with the FY1s was not enough. Consequently, any further cycles will require more concrete protocol based interventions. Further cycles of the project may include implementing a step in the preoperative ward admission protocol to require nursing staff to contact the team's doctor to complete the drug chart documentation. Discussions with the pharmacy after the project revealed some important points. For example, there is already a high risk group of medications that must not be omitted and that using these medications would have been more appropriate. Multidisciplinary team discussions prior to the next cycle would improve the project's credibility. 


\section{BMJ Quality Improvement Reports}

\section{Conclusion}

Following the cancellation of FY1 preoperative clinics, awareness of a crucial responsibility was lost. Patients undergoing elective surgery were being admitted to surgical wards postoperatively without medication reconciliation and adequate drug chart documentation.

A primary medication history should be taken for every patient admitted to hospital by a member of the admitting team, and their drug chart adequately completed, including allergy status, regular medications and thromboprophylaxis. Interventions designed to empower the FY1 doctors and prompt them to fulfill this role resulted in improved standards in correct drug chart prescriptions and allergy status documentation. However, the impact of the interventions relied on FY1s fulfilling these responsibilities simply by being made aware of the associated risks and this project shows that this has not been enough.

Ultimately more needs to be done to ensure accurate medicine reconciliation on the day of admission to hospital. This might include implementing a step within the patient admission protocol in the preoperative ward that requires the nursing staff to contact the team's doctor when the patient arrives at hospital to ensure satisfactory drug chart documentation. In addition, collaboration with the pharmacists could also allow a BPMH to be taken on the day of admission and thus reduce risk to patient safety.

\section{References}

1. National Institute for Health and Care Excellence (NICE) Guidelines. Technical patient safety solutions for medicines reconciliation on admission of adults to hospital (PSG001).

2. Van der Schrieck-de Loos E, van Groenestijn A. High 5's Med Rec SOP. International standard operating procedure for medication reconciliation in the Netherlands. 2011

3. Gleason KM, McDaniel MR, Feinglass J, et al. Results of the Medications At Transitions and Clinical Handoffs (MATCH) study: an analysis of medication reconciliation errors and risk factors at hospital admission. J Gen Intern Med 2010;25:441-7

\section{Declaration of interests}

Nothing to declare.

\section{Acknowledgements}

Dr G Mennon, Director of Medical Education, Croydon University Hospital 